Florence Gacoin-Marks

Faculté des lettres

Université de Ljubljana

Slovénie

florence.gacoin-marks@guest.arnes.si
UDK 81'255.4=133.1=163.6:82-31Houellebecq M. DOI: $10.4312 /$ vestnik.13.365-381

\title{
TRADUCTION DES CONNOTATIONS AUTONYMIQUES DANS TROIS ROMANS DE MICHEL HOUELLEBECQ
}

Très commentée et étudiée pour ses thématiques, l'œuvre de Michel Houellebecq n'a donné lieu qu'à un nombre relativement restreint d'études stylistiques. ${ }^{1}$ D'abord, parce que le romancier a été maintes fois qualifié d'écrivain « sans style», ce qui est assez inhabituel pour être souligné. ${ }^{2}$ Puis en raison du fait que son œuvre a été tant commentée pour son contenu sociétal, provocateur, voire idéologique que l'on en a presque oublié que nous sommes face à une œuvre fictionnelle, comme ne cesse de le répéter son auteur dans ses interviews. ${ }^{3}$

Et pourtant, il y a bien un style houellebecqien. Parmi les signes le caractérisant, un phénomène saute aux yeux dès la première lecture : la présence d'un nombre non négligeable d'éléments textuels (50 éléments par 100 pages en moyenne) signalés dans le texte par un usage de l'italique ou des guillemets ne relevant pas de l'utilisation codifiée de ces deux moyens typographiques de mise en relief. ${ }^{4}$ Le premier à avoir attiré l'attention sur l'importance de ce stylème dans l'œuvre de Houellecbeq est son éditeur et ami Dominique Noguez, auteur de l'un des premiers livres entièrement consacrés à l'écrivain (2003, 129-132). Mais il s'agit pour ce dernier davantage de prouver que le style de Houellebecq, souvent présenté comme inexistant, est en réalité beaucoup plus subtil qu'il n'y paraît. Il est ensuite mentionné par de nombreux chercheurs, sans toutefois avoir fait l'objet d'une étude systématique prenant également en compte l'évolution de l'écriture houllebecquienne depuis les années 1990 jusqu'à nos jours. ${ }^{5}$

Véritable stylème clairement identifié par les lecteurs comme par les chercheurs, l'utilisation élargie de l'italique et des guillemets est donc un phénomène sur lequel le lecteur est en droit - et le traducteur en devoir - de s'interroger pour décoder sa signification

1 Voir Jafuel 2016: 4-10.

2 Sur l'ensemble des polémiques autour du style de Houellebecq, voir Estier (2015).

3 Voir notamment les interviews données à France Inter et RTL au moment de la promotion de Soumission en 2015.

4 Voir les nombreuses grammaires « de référence », notamment Riegel [et al.] 1994, chapitre IV, ainsi que les différents usuels destinés aux relecteurs (entre autres, le Lexique des règles typographiques en usage à

l'Imprimerie nationale, 2002.

5 Voir Jafuel 2016: 15. 
exacte. ${ }^{6}$ Le corpus de trois romans servant de base à la présente étude traductologique a été constitué de manière à inclure des traductions élaborées par trois traducteurs slovènes différents : Marko Trobevšek, Mojca Medvedšek et Iztok Ilc, trois traducteurs diplômés de langue française et expérimentés dans le domaine de la traduction de la littérature française contemporaine. ${ }^{7}$ Nous pourrons ainsi examiner quelle stratégie chacun d'entre eux a adopté pour traiter les éléments analysés.

En littérature, les éléments signalés par l'italique ou les guillemets (sans être du discours direct stricto sensu) ont tous une fonction métalinguistique, car ils connotent les signes linguistiques comme tels, tout en continuant à dénoter le monde représenté dans le récit (Rey-Debove, 1979, 33). C'est la raison pour laquelle, nous pouvons les appeler, en accord avec la terminologie adoptée par les linguistes et sémioticiennes françaises Josette Rey-Debove et Jacqueline Authier-Revuz, des « connotations » ou « modalisations » autonymiques. ${ }^{8}$ Ce sont les éléments que le narrateur met visuellement à distance, dont il se démarque du fait qu'ils sont, d'une manière ou d'une autre, étrangers à son discours (signes d'idiolectes particuliers ou de sociolectes collectifs). L'italique et les guillemets sont donc ici des marqueurs d'hétérogénéité discursive.

Sur le plan narratif, le recours à la modalisation autonymique - qui devient ici un stylème - a l'avantage considérable de ne pas interrompre le récit, d'introduire des nuances « en passant », sans ralentir le flux narratif. En effet, comme l'a souligné Gregor Perko :

« [L]a modalisation autonymique, qui met en relief la 'matérialité' du signe linguistique, ne présente nullement un dysfonctionnement de la communication qui provoquerait une rupture de la progression thématique, bien que 'l'arrêt-sur-langue' ou 'l'arrêt-sur-énonciation' entraîne une sorte de 'décrochage' communicatif. Plus précisément, l'énoncé se 'dédouble' et se développe à deux niveaux distincts : un niveau mondain et un niveau métalinguistique » (2012: 255).

Il s'agit donc d'un stylème puissant sur le plan sémantique, mais aussi très efficace sur le plan narratif. Comme l'a noté Jacqueline Authier-Revuz,

« les discours qui circulent concurremment, se répondent, s'épaulent, s'affrontent, se démarquent..., dans le même 'champ' que le mien - des discours

6 Comme l'écrit Jacqueline Michel à propos de ce stylème chez Julien Gracq, c'est un procédé qui « interroge et demande à être interrogé » (1981:14).

7 Mojca Medvedšek a également traduit Plateforme, La Possibilité d'une île, La Carte et le territoire et Soumission.

8 Rey-Debove, Josette (1997) ; Authier-Revuz (1978, 1992-1993, 1996, 2029). De manière générale, cette dernière répartit les formes de discours rapporté en trois catégories : les formes marquées et univoques, les formes marquées exigeant un travail interprétatif (segments relevant d'une modalisation autonymique marqués par des guillemets, par des italiques ou à l'oral par l'intonation) et les formes purement interprétatives (discours direct libre, discours indirect libre, citations cachées, allusions). Le présent article porte en majeure partie sur la seconde catégorie (1992, n 55, 41-42). 
qui, avant moi, et comme moi, mieux que moi, pas comme moi, pas tout à fait comme moi, pas du tout comme moi,... ont parlé de ce dont je parle, discours que 'croise', inévitablement, mon discours et par rapport auquel il se 'positionne' » (1996: 113).

Avant d'analyser la traduction des éléments signalés en italique et entre guillemets par Houellebecq, il convient de préciser que nous nous sommes ici limités aux utilisations de l'italique et des guillemets non exigées par la norme typographique. ${ }^{9}$ Ont donc été exclus les citations d'auteurs, les titres et les mots étrangers qu'il est impossible d'écrire sans les signaler par l'italique ou les guillemets. ${ }^{10}$ Il est également important de noter qu'il n'existe pas de différence notable entre les langues française et slovène en ce qui concerne les usages de l'italique et des guillemets prescrits par la norme orthographique et ceux relevant de la mise en relief. Il est donc théoriquement possible d'avoir recours à ces deux procédés pour aboutir en slovène aux effets recherchés par l'écrivain français. ${ }^{11}$

Dans les pages suivantes sont présentées les principales catégories d'occurrences du stylème ainsi que des remarques concernant leur traitement par les trois traducteurs slovènes de l'écrivain français. ${ }^{12}$

\section{LES « ILÔTS TEXTUELS », LA PAROLE DE L'AUTRE AU SEIN DU RÉCIT DU NARRATEUR}

L' « îlot textuel », ${ }^{13}$ le signalement au sein même du récit d'une parole étrangère à celle du narrateur, est en quelque sorte l'usage « historique », le plus ancien dans la

9 Sur les usages élargis de l'italique et des guillemets en littérature, on peut prendre pour point de départ fournissant le lien entre sémiotique, linguistique et stylistique l'ouvrage d'Anne Herschberg Pierrot (1993). Par ailleurs, pour les éléments analysés, il ne sera pas fait de distinction entre l'usage de l'italique et celui des guillemets. Intéressante au demeurant, cette question n'influe pas sur le traitement des autonymes par les traducteurs et n'a donc pas lieu d'être abordée dans le présent article.

10 Cela ne signifie cependant pas que la mise en relief des noms de lieux ou des titres n'a pas, comme chez Flaubert, une double signification autonymique : celle de signaler le titre et celle de mettre en valeur une expression ayant du sens dans une situation donnée. Comme ici : "J'eus l'étrange impression de pénétrer dans une sorte d'autofiction en pénétrant dans la salle des pas perdus de la gare Saint-Lazare » (Houellebecq 2019 : 157). Le nom de lieu entre en résonnance avec la situation existentielle du narrateur. Cette référence s'est totalement perdue dans la traduction slovène (Houellebecq - Ilc 2020 : 136).

11 La section explicative « Pravila » de la dernière édition du manuel orthographique normatif Slovenski pravopis (https://fran.si/134/slovenski-pravopis/datoteke/Pravopis_Pravila.pdf) ne mentionne pas l'usage de l'italique hormis celui permettant la mise en relief des titres. L'usage « expressif » est donc totalement laissé à l'appréciation de l'auteur. En revanche, on y trouve toute une section consacrée aux guillemets où l'auteur distingue les usages syntaxiques et non syntaxiques. C'est dans la seconde section que sont regroupés les usages faisant l'objet de la présente analyse.

12 Notons qu'il y a inévitablement une part de subjectivité dans le classement des exemples proposés. Mieux encore, les catégories ne sont pas strictement séparées les unes des autres. En effet, comme déjà évoqué, cette utilisation est parfois volontairement équivoque et admet plusieurs interprétations qu'il conviendra de prendre en compte.

13 L'appellation d'« îlot textuel » est de Jacqueline Authier (1978, puis 1996). Elle définit ce phénomène comme la « représentation d'un discours autre », c'est donc un autre mot pour la modalisation autonymique explicite. 
littérature française, celui que Balzac emploie souvent pour rapporter des expressions régionales ou autrement connotées de ses personnages. ${ }^{14}$ Ce n'est pas l'usage le plus fréquent chez Houellebecq et il est presque toujours signalé par un commentaire autonymique explicite.

\subsection{La parole d'un locuteur clairement défini}

Parfois, la parole revient à une personnalité clairement identifiée. La citation du vocabulaire précis utilisé et la mise en relief de ce dernier par les guillemets ou l'italique replacent le lecteur dans un contexte historique, dans l'esprit d'une époque, comme ici :

« Sur le plan professionnel, son seul objectif était - très raisonnablement - de se fondre dans cette 'vaste classe moyenne aux contours peu tranchés' plus tard décrite par le président Giscard d'Estaing » (Houellebecq 1998 : 64).

« Na poklicnem področju je bil njegov edini cilj - in to zelo razumen - stopiti se z 'mnogoštevilnim srednjim razredom z zabrisanimi mejami', kot ga je označil predsednik Giscard d'Estaing » (Houellebecq - Medvedšek 2000 : 65).

Il arrive aussi que l'attention soit portée sur les mots caractéristiques d'une parole plus collective, invitée dans le récit pour sa « couleur locale ", son caractère pittoresque, comme ici où il est question des vaches qui vont « comme le disent les éleveurs dans leur parler cynique, 'se faire remplir' » (Houellebecq 1994 : 10), ce que le traducteur a très justement traduit par « kot govorijo rejci v svoji posmehljivi govorici, 'biti napumpana' » (Houellebecq - Trobevšek $2004: 10$ ).

Dans d'autres cas, plus fréquents, les citations sont clairement attribuées à des personnages romanesques qui s'invitent ainsi dans la narration principale. Attirer l'attention sur les mots exacts qu'ils emploient permet à l'écrivain de les caractériser discrètement sans interrompre son récit par des gloses descriptives concernant leur psychologie :

« [Tisserand] m'a alors expliqué qu'un reste d'orgueil l'avait toujours empêché d'aller aux putes » (Houellebecq 1994 : 99).

« Potem mi je [Tisserand] razložil, da zaradi ostanka ponmosa nikoli ni mogel iti h kurbam » (Houellebecq - Trobevšek 2004 : 78).

L'utilisation de cette expression souligne le fond d'idéalisme qui caractérise ce personnage par rapport au narrateur : encore convaincu qu'il est possible de construire une relation sentimentale avec l'autre sexe, le personnage affiche son mépris pour les amours

14 Voir notamment dans Les Paysans et Splendeur et misère des courtisanes. 
vénales. Comme bien souvent, la parole du personnage s'apparente à la langue parlée, voire vulgaire, de sorte que l'usage de l'italique pourrait simplement signaler la distance entre l'expression du narrateur et celle du personnage. Mais l'effet produit est double. Il en est de même dans l'exemple suivant :

« En bref, et comme Bruno le résuma une fois à l'intention de son psychiatre, 'tout se barrait en couille' » (Houellebecq 1998 : 77).

« Skratka, kakor je to Bruno nekoč na kratko opisal svojemu psihatru, 'vse gre prej ali slej v maloro’ » (Houellebecq - Medvedšek 2000, 77).

Dans son désarroi, Bruno s'exprime en termes crus, sa phrase sonne presque comme un juron dénonçant le caractère désespéré de la situation (et peut faire sourire le lecteur s'attendant à une réflexion plus élaborée). L'expression slovène n'est pas inadéquate, mais il lui manque la vulgarité de l'expression française. L'expression ordurière « vse gre prej ali slej v kurac » aurait mieux collé à la peau de Bruno.

Même emploi ici, où la mention caractérise le personnage de Claire, une femme moderne qui n'éprouve pas de gêne à parler de sa sexualité, y compris en employant des expressions crues :

« [...] il aurait pu lui arriver n'importe quoi d'autant qu'elle commençait sérieusement, selon ses propres termes, à 's'intéresser à la bite' » (Houellebecq 2019 : 129).

« [...] lahko bi se ji pripetilo karkoli, še toliko bolj, ker se je po njenih besedah začela resno 'zanimati za tiče' » (Houellebecq - Ilc 2020 : 112).

La vulgarité caractérisant le personnage et expliquant la mention par le narrateur est tout à fait bien rendue par l'expression slovène choisie par le traducteur. ${ }^{15}$

\subsection{Une parole autre, mais opaque}

Dans de nombreux cas, la parole d'autrui est rapportée sans être clairement identifiée. Par exemple ici :

« D’emblée, je l'informe que je suis en dépression » (Houellebecq 1994: 135). «Takoj ga obvestim, da sem v depresiji » (Houellebecq - Trobevšek 2004: 110).

15 Comme en témoignent certains écarts sémantiques, notamment dans la traduction des Particules élémentaires, ces expressions sont parfois difficiles à identifier par un traducteur n'étant pas en contact régulier avec le français parlé familier, voire ordurier. 
S'agit-il du diagnostic posé par le psychiatre ou de l'expression " générique » utilisée par le narrateur pour qualifier son mal-être ? Dans ce cas simple, cela n'a aucune incidence sur la traduction, qui sera la même dans les deux cas.

De même dans l'exemple suivant :

« $[\mathrm{U}] \mathrm{n}$ défilé a eu lieu à Paris pour protester contre les 'brutalités policières' ; il s'est déroulé dans une atmosphère 'd'une dignité bouleversante' (Houellebecq $1994: 61)$.

« [Bil] je v Parizu sprevod v protest proti 'policijski surovosti'; potekal je v ozračju 'ganljive dostojanstvenosti' (Houellebecq - Trobevšek 2004: 50).

Les cas de ce type sont très nombreux et, comme pour les citations clairement identifiées, les traducteurs slovènes ont généralement cherché et trouvé des solutions appropriées pour les traduire en conservant l'emploi de l'italique ou des guillemets.

Parfois, pour diverses raisons, le narrateur met à distance des éléments de son propre discours. Il ne s'agit donc pas d' "îlots textuels » au sens strict, mais d'éléments considérés comme étrangers par le narrateur du fait qu'il ne les assume pas ou qu'il émet de sérieuses réserves à leur égard.

\subsection{L'utilisation d'expressions crues, voire vulgaires}

Cet emploi peu fréquent est, en un sens, proche de l'emploi orthographique : il s'agit pour le narrateur de signaler les expressions extérieures à la langue standard mais qui confèrent au récit plus d'authenticité et de pittoresque. Comme nous avons déjà vu un cas similaire s'agissant des expressions vulgaires utilisées par certains personnages (et signalées la plupart du temps entre guillemets), il est inutile de nous attarder outre mesure. Les expressions vulgaires devront être identifiées, puis traitées systématiquement : s'il existe en slovène un équivalent adéquat sur le plan sémantique et stylistique, il pourra être signalé en italique comme dans le texte original. Dans le cas où elles seront remplacées par des traductions sémantiquement adéquates mais ne relevant pas du registre familier ou vulgaire, il faudra renoncer à l'emploi de l'italique. Ainsi, dans les deux exemples suivants :

« Généralement, il racontait des histoires de cul; je sens que ce déplacement en province va être sinistre (Houellebecq $1994: 52$ ). 
«V glavnem je pripovedoval kosmate zgodbe; glede te poti na deželo me obhajajo zle slutnje (Houellebecq - Trobevšek 2004 : 44).

Ici, le traducteur a trouvé un équivalent slovène qui, bien que moins vulgaire que l'expression française, introduit du pittoresque dans la langue du narrateur. D'où la conservation de l'italique. Il en est autrement dans l'exemple suivant :

« Son oncle votait toujours communiste et refusait d'aller à la messe de minuit, c'était à chaque fois l'occasion d'un coup de gueule » (Houellebecq 1998 : 155). « Njegov stric je volil komuniste in ni hotel iti k polnočnicam, kar je vsako leto znova povzročilo glasno zmerjanje » (Houellebecq - Medvedšek 2000 : 151).

N'ayant pas trouvé d'expression aussi familière et imagée en slovène, la traductrice a donc opté pour un équivalent standard descriptif (adjectif + substantif) qu'elle n'a pas jugé opportun de signaler en italique. ${ }^{16}$

Toutefois, on remarque des écarts montrant que tous les traducteurs sont conscients de la difficulté mais qu'aucun d'entre eux n'a véritablement élaboré une stratégie cohérente.

Ainsi, dans la traduction de L'Extension du domaine de la lutte, nous lisons :

« Je n'éprouvais aucun désir pour Catherine Lechardoy ; je n'avais nullement envie de la troncher [...] ; pourtant, je le savais, elle avait tellement besoin d'être tronchée » (Houellebecq 1994 : 46).

« Do Catherine Lechardoy nisem občutil nikakršnega poželenja; niti malo mi ni bilo do tega, da bi jo nategnil » (Houellebecq - Trobevšek 2004: 37).

Ici, le traducteur a trouvé un parfait équivalent (tout aussi vulgaire qu'en français) qu'il a mis en italique. En revanche, l'usage du même mot au passif lui ayant probablement semblé lourd en slovène, il a décidé de purement et simplement supprimer la dernière remarque du narrateur, ce qui est dommageable pour le texte, alors qu'il aurait suffi d'écrire une formule elliptique permettant de conserver l'idée exprimée sans alourdir le style (« čeprav je to še kako potrebovala », « čeprav bi ji to še kako koristilo », ipd.).

Même constat dans l'exemple suivant, extrait de Sérotonine : « [I]1 demeurait sans doute encore des queutards et des baiseuses » (Houellebecq 2019 : 323). Ici, le narrateur emploie deux expressions très vulgaires pour désigner les hommes et les femmes qui enchaînent les relations sexuelles : Or, en slovène, nous lisons : « najbrž so še vedno obstajali potrebneži in fukači » (Houellebecq - Ilc 2020 : 280). Cette traduction est

16 Dans un cas similaire, le traducteur de Sérotonine a conservé l'italique qui, ne signalant pas une expression familière, se trouve vidé de son sens : « J'avais moi-même changé, physiquement, j'étais conscient que j'avais subi un ou plusieurs coups de vieux » (Houellebecq 2019 : 246) ; «Jaz pa sem se telesno spremenil, jasno mi je bilo, da se se pošteno postaral » (Houellebecq - Ilc 2020 : 245). 
problématique du point de vue tant sémantique que stylistique. Tout d'abord, le texte slovène ne mentionne que les hommes, ce qui n'est pas le sens de l'original qui parle bien des deux sexes. Par ailleurs, si l'expression « fukači » est bien ordurière, en revanche « potrebneži » ne l'est pas, ce qui remet fortement en cause l'usage de l'italique.

\subsection{L'utilisation d'expressions orale à la mode}

Même cas que le précédent, mais pour des expressions laissant transparaître discrètement la société et l'époque dans laquelle le narrateur évolue. Le traducteur aura donc cette fois pour tâche de trouver un équivalent parmi les expressions à la fois équivalentes sur le plan sémantique et caractéristiques du slovène contemporain, ce qui est rarement chose aisée. Examinons quelques exemples :

(a) Une bonne boîte

«à tous points de vue, une bonne boîte ( Houellebecq1994: 17).

« v vseh pogledih solidna družba » (Houellebecq - Trobevšek $2004: 16$ ).

(b) [L]e zéro défaut

« nous étions encore loin du zéro défaut » (Houellebecq 1994 : 45);

« smo še daleč od brezhibnosti » (Houellebecq - Trobevšek $2004: 36$ ).

(c) « un message subtilement second degré» (Houellebecq $1998: 23$ ); dvomljivo sporočilo (Houellebecq - Medvedšek 2000 : 23)

(č) « [J]e n'avais pas été formaté pour une telle proposition, ça ne faisait pas partie de mon logiciel » (Houellebecq 2019: 172);

«Vendar tega nisem storil, in tega najbrž nisem mogel, za takšen predlog nisem bil formatiran, to ni bil del moje programske opreme » (Houellebecq-Ilc : 148).

L'exemple (a) relève du monde du travail et désigne familièrement une entreprise, une « boîte », qualifiée de « bonne » dans le sens où elle a de bons résultats et traite correctement ses employés. « Solidna firma » (au lieu du syntagme inhabituel « solidna družba » que le traducteur n'a pas, à juste titre, écrit en italique) aurait été un meilleur équivalent qui aurait pu, lui, être signalé en italique au même titre que l'original français. Dans les exemples (b) et (c), ce sont des caractéristiques syntaxiques qui signalent les expressions à la mode : l'élipse « le zéro défaut » au lieu de « quelque chose n'ayant aucun défaut » et le « message second degré » au lieu du «message au second degré ». Ne pouvant trouver d'équivalent sémantique ayant les mêmes connotations en slovène, les deux traducteurs 
ont opté par une variante standard non connotée qu'ils n'ont logiquement pas mis en valeur par l'emploi de l'italique. L'exemple (d) rapporte l'usage métaphorique fréquent des mots « formaté » et « logiciel » empruntés au domaine de l'informatique pour désigner la manière de penser d'un personnage. La révolution technologique étant mondiale, ces deux mots sont parfaitement traduisibles et revêtent en slovène la même signification et connotation qu'en français, d'où l'usage de l'italique, parfaitement justifié dans ce cas.

\subsection{Mise en relief d'expressions banales ou stéréotypées}

Cette utilisation assez fréquente est particulièrement intéressante du point de vue traductologique. En effet, par la mise en relief à l'aide de l'italique ou des guillemets, l'auteur engage le lecteur à envisager certaines expressions du français courant sous un angle nouveau. Il les dégage des automatismes pour permettre leur réinterprétation. ${ }^{17}$

Dans certains cas, il s'agit juste d'attirer l'attention du lecteur sur elles :

« Elle avait trouvé un peu bizarre que Michel rentre avant les autres, oui ; elle avait également trouvé bizarre qu'il parte s'installer un mois avant la rentrée universitaire, mais Michel était un garçon bizarre » (Houellebecq 1998 : 82).

« Čudno se ji je zdelo le, da se je prvi vrnil s počitnic; še bolj čudno se ji je zdelo, da se je naselil v študentskem naselju mesec dni pred ostalimi; toda Michel je bil čudak » (Houellebecq - Trobevšek : 87).

« Tout cela était atrocement réel » (Houellebecq 1998: 115) ; « Bref, là encore, on assistait à un authentique moment de vie réelle » (118).

« In vse to, kar se je dogajalo, je bilo kruto resnično » (Houellebecq - Medvedšek 2000 : 113) ; «Skratka, tudi tukaj je bil človek priča trenutkom resničnega Življenja » (115).

« Et avais-je vraiment envie de savoir ce que Claire était devenue ? (Houellebecq 2019 : 117).

« In ali sem si res želel vedeti, kaj je Claire postala? » (Houellebecq - Ilc 2020 : 100).

Ici, le narrateur insiste sur le fait qu'il convient de comprendre l'expression banale dans son sens plein, fort. Dans les deux premiers exemples, l'expression n'est ni imagée ni figée ou stéréotypée, l'équivalence coule donc de source. Et pourtant le traducteur de

17 C'est ce qui participe à ce que Christèle Couleau appelle le « réalisme de défamiliarisation » où « ce qui semblait familier prend un caractère nouveau d'étrangeté, un relief inattendu » (2013:16). 
L'Extension du domaine de la lutte n'a pas jugé nécessaire de souligner le verbe biti («je ») à l'aide de l'italique comme l'a fait Houellebecq avec le verbe « être ». Dans le troisième exemple, pourtant simple en apparence, se profile déjà le cœur du problème : le traducteur est contraint d'utiliser la traduction littérale de « devenir » (« postati »), beaucoup moins banale en slovène qu'en français et de faire ainsi ressortir le sens plus fort sous-entendu par l'italique. ${ }^{18}$ Peu perceptible ici, cette difficulté devient plus grande dans l'exemple suivant:

"Peut-être, me dis-je, ce déplacement en province va-t-il me changer les idées; sans doute dans un sens négatif, mais il va me changer les idées; il y aura au moins un infléchissement, un soubresaut» (Houellebecq 1994 : 49).

«Morda, si pravim, me bo pot po podeželju spreobrnila; nedvomno na slabše, vendar se bom malo spreobrnil; vsaj do odklona bo prišlo, do trzaja (Houellebecq - Trobevšek 2004: 38).

Dans la langue courante, « se changer les idées » signifie se reposer du stress quotidien, se divertir ; en slovène, on pourrait dire « razvedriti » ou « se oddahniti ». Mais en ayant recours à l'italique, le narrateur montre que l'expression doit être également comprise dans un sens plus fort, comme un synonyme de « changer sa façon de penser, d'envisager l' existence ». D'où ce choix du verbe « spreobrniti se » (« se convertir »), qui est une solution maladroite du fait qu'il est trop fort et ne relève pas du tout du langage courant, ce qui rend caduque l'usage de l'italique. LA SOCIÉTÉ CONTEMPORAINE OU DE LA « SOCIÉTÉ D'AVANT »

Cette fonction des modalisations autonymiques est la plus répandue chez Houellebecq. C'est aussi celle où le recours à la connotation ou modalisation autonymique joue le rôle le plus important dans le récit. On peut même dire que, mis bout à bout, ces éléments propose aux lecteurs une image synthétique de la société dans laquelle le personnage évolue. Or, celle-ci est comme mise à distance par un narrateur " postréaliste » cherchant non pas seulement à décrire et/ou à dénoncer, mais à faire prendre conscience au lecteur des traits caractéristiques de la société dans laquelle il vit (dans Extension du domaine de la lutte et Sérotonine) ou de la société antérieure, dont l'humanité a réussi à s'extraire en évoluant vers une nouvelle forme de vie (dans Les Particules élémentaires). ${ }^{19}$

18 Pour dire « Qu'est-ce que X devient/est devenu ? », on dira plutôt « Kaj je (bilo) z njim? » ou « Kako mu gre? », «Kako kaj življenje? » etc.

19 En ce sens, nous rejoignons l'analyse de Sabine Wesemael qui, après avoir étudié ce qui rapproche Houellebecq du postmodernisme, parvient finalement à la conclusion qu'il est plus juste de définir l'écrivain comme postréaliste : « Houellebecq est avant tout l'analyste sceptique du sort absurde, irrésolu, de l'homme contemporain » $(2013,336)$. 


\subsection{Normes et canons de la société contemporaine}

D'autres occurrences de l'italique et des guillemets servent à signaler les normes et canons de la société dans laquelle le narrateur évolue. Dans les deux exemples suivants, par exemple, l'objectif est de montrer l'inadéquation entre ces normes et la vie du narrateur :

« je serai rentré pour Noël, afin de me permettre de 'passer les fêtes en famille’ (Houellebecq 1994 : 39)

« nazaj bom za božič, da bom lahko preživel praznike z 'družino' » (Houellebecq-Trobevšek $2004: 30-31$ )

« Lors de mon retour 'à la maison' » (Houellebecq 1994 : 104).

« Ko sem se vrnil 'domov’ ( Houellebecq - Trobevšek 2004 : 82).

Dans le premier cas, le narrateur souligne l'incongruité, voire l'absurdité de l'expression « passer les fêtes en famille » dans le cas d'un homme comme lui, célibataire et sans famille. Même chose pour l'expression «à la maison », qui n'a pas le même sens pour le narrateur que pour la majorité de ses contemporains.

Dans d'autres exemples, le narrateur cherche à faire ressortir le triomphe du « tout économique » au cœur de L'Extension du domaine de la lutte et ses ramifications dans la vie amoureuse et sexuelle des hommes de la fin du $\mathrm{XX}^{\mathrm{e}}$ siècle. C'est le cas ici :

« le libéralisme sexuel produit des phénomènes de paupérisation absolue » (Houellebecq 1994 : 100).

« spolni liberalizem povzroča popolno obubožanje » (Houellebecq - Trobevšek 2004 : 79).

Cette intrusion du vocabulaire économique jusque dans la vie intime des êtres humains n'est pas signalée en italique par le traducteur slovène.

Dans la logique de ce qui vient d'être écrit, les clés de la séduction sont soit dans le « charme » et la « classe », soit dans l'argent :

« [I]l n'a absolument aucun charme (le charme est une qualité qui peut parfois remplacer la beauté - au moins chez les hommes ; d'ailleurs on dit souvent : 'Il a beaucoup de charme', ou : 'Le plus important, c'est le charme'; c'est ce qu'on dit) » (Houellebecq1994: 54).

« prav nobenega čara nima (čar je vrnila, ki včasih lahko nadomesti lepoto vsaj pri moških; sicer pa pogosto pravijo: 'zelo očarljiv je' ali: 'za čar gre'; tako pravijo)» (Houellebecq - Trobevšek $2004: 45)$. 
« [T] ous ces gens n'avaient définitivement aucune classe, et d'ailleurs moi non plus je n'avais aucune classe, seulement j'avais de l'argent, pas mal d'argent même » (Houellebecq $2019: 22$ ).

« vsi ti ljudje so bili definitivno brez vsakega stila, sploh pa tudi jaz nisem imel nobenega stila, imel sem samo denar, [...] niti ne tako malo denarja » (Houellebecq - Ilc $2020: 18)$.

Les deux traducteurs ont signalé l'expression en italique, et pourtant leur traduction n'est pas équivalente : dans la première, l'expression « avoir du charme » est traduite trop littéralement (« ne imeti čara » est peu naturel et peut même être considéré comme un gallicisme); le traducteur a dû s'en rendre compte lui-même, car il a par la suite remplacé cette expression par « zelo očarljiv je » et « za čar gre ». ${ }^{20}$ En revanche, dans la seconde traduction, « imeti slog » est un équivalent très proche de l'expression française « avoir de la classe ».

\subsection{Misères de la société « d'avant » vue par les néo-humains}

Cette catégorie n'apparaît que dans Les Particules élémentaires qui s'avère être un roman d'anticipation, raconté par un «néo-humain » dont l'objectif est de faire la propagande de la nouvelle société « posthumaine » et donc de présenter la vie de l'homme de la fin $\mathrm{du} \mathrm{XX}^{\mathrm{e}}$ siècle comme terrifiante, insupportable pour l'homme et résolument inapte à lui apporter le bonheur. Le narrateur doit donc expliquer à son lecteur « néo-humain » quels étaient les concepts caractéristiques de la vie humaine avant sa chute, avant sa transformation radicale.

Par exemple, la vie de famille à la fin du $\mathrm{XX}^{\mathrm{e}}$ siècle rend souhaitable, voire nécessaire, la vie dans un environnement moins exigu et bétonné que les grandes métropoles, une sorte de compromis entre la ville où l'on travaille et la campagne où on a de l'espace pour vivre :

« Les couples sont fidèles et heureux ; ils vivent dans des maisons agréables en dehors des villes (les banlieues)» (Houellebecq $1998: 49$ ).

«Zakonski pari so si zvesti in srečni; živijo v prijetnih hišah izven mesta » (Houellebecq-Medvedšek 1998 : 50).

La traductrice a jugé que l'équivalent slovène « predmestje » ne reflétait pas la même réalité en Slovénie que le mot «banlieue » en France, la notion mise en relief par l'italique. Elle l'a donc omis.

20 Solution alternative : «Prav nič šarmaten ni bil ». 
Un autre concept de la « vie d'avant» est la réussite personnelle et sociale traduite par deux expressions être « arrivé » et « avoir réussi sa vie » :

« À quarante-deux ans, Serge Clément était un homme arrivé » (Houellebecq 1998: 47).

« Pri dvainštiridesetih letih je bil Serge Clément prišlek» (Houellebecq-Medvedšek $2000: 49$ ).

« Fils d'un anarchiste italien émigré aux États-Unis dans les années vingt, Francesco di Meola avait sans nul doute réussi sa vie, sur le plan financier s'entend » (Houellebecq 1998: 80).

«Francesco di Meola je bil sin italijanskega anarhista, ki je v dvajsetih letih emigriral v Združene države. Brez dvoma mu je v finančnem pogledu v življenju uspelo » (Houellebecq - Medvedšek 2000 : 80).

Dans la première occurrence, la traductrice a cherché une expression slovène équivalente, mais a malheureusement modifié le sens de l'expression : " prišlek » ne signifie pas « arrivé » mais « nouvel arrivant », « nouvellement arrivé dans un pays ou une région », « immigré ». L'expression « (dobro) situiran » aurait permis de préserver et le sens de l'expression et l'italique soulignant la référence à l'une des notions cardinales de la société, notamment de celle de la fin du $\mathrm{XX}^{\mathrm{e}}$ siècle, comme c'est le cas dans le second exemple, où la traduction est parfaitement conforme au texte original.

L'usage de ce type le plus fréquent concerne bien entendu le point le plus litigieux de la transformation de l'homme en néo-humain (et, de ce fait, le cœur de la propagande néo-humaine) : la modification radicale des relations entre les hommes et les femmes. Ainsi, pour montrer comment fonctionnait la société « d'avant », le narrateur néo-humain met en évidence grâce à l'italique les concepts désormais disparus. Observons quelques exemples :

« [...] du premier coup, sans l'avoir cherché, sans même l'avoir réellement désiré, elle se trouvait en présence du grand amour » (Houellebecq 1998 : 56). « Ne da bi jo prav posebej iskala, ne da bi si jo v resnici želela, se je znašla pred veliko ljubeznijo » (Houellebecq - Medvedšek 2000 : 58).

« déclin du mariage de raison » (Houellebecq 1998: 53) ; « évolution vers le mariage d'amour » (54)

« zaton dogovorjenih porok; nagnjenost k poroki iz ljubezni » (HouellebecqMedvedšek 2000 : 55). 
«Les jeunes gens des années cinquante attendaient de tomber amoureux » (Houellebecq $1998: 54$ ).

«Mladi so v petdesetih letih torej [...] komaj čakali, da se zaljubijo » (Houellebecq-Medvedšek $2000: 55$ ).

Nous constatons que, dans ce cas, la traductrice a respecté l'emploi houellebecquien de l'italique.

Dans le cadre d'un style souvent qualifié de « blanc », l'usage élargi de l'italique et des guillemets à fonction de connotation autonymique permet donc à l'auteur de transmettre au lecteur des nuances sémantiques d'une grande finesse et concoure à la richesse de son œuvre. Comme les réseaux métaphoriques, les stylèmes tel que l'emploi récurrent et élargi de l'italique et des guillemets, exigent du traducteur un travail d'interprétation préalable et l'élaboration d'une stratégie cohérente permettant de préserver intacte sa fonction dans le récit. Bien que les trois traductions analysées puissent être considérées comme globalement fidèles au texte de Houellebecq et aient rendu possible la notoriété de l'écrivain français en Slovénie, force est de constater qu'aucun d'entre elles ne se distingue par un traitement rigoureux et cohérent des connotations autonymiques signalées par un emploi non normatif de l'italique et des guillemets. Le maintien de ces signes visuels là où les expressions signalées en français n'ont pas lieu de l'être en slovène ou, au contraire, leur suppression là où il est parfaitement possible et opportun de les maintenir sont les anomalies les plus fréquentes et dommageables pour la compréhension fine du texte. Notons qu'il pourrait être intéressant à l'avenir d'étudier de manière plus globale l'évolution de l'usage de l'italique et des guillemets dans l'œuvre de Houellebecq ${ }^{21}$ et d'évaluer en quoi cette évolution devrait être prise en compte dans l'élaboration des traductions, et ce quelle que soit la langue cible envisagée. ${ }^{22}$

\section{BIBLIOGRAPHIE}

\section{Primaire}

\section{Textes originaux (français)}

HOUELLEBECQ, Michel (2003) Extension du domaine de la lutte. Paris : J'ai lu [1": 1994]. HOUELLEBECQ, Michel (2003) Les Particules élémentaires. Paris : J'ai lu [1¹998]. HOUELLEBECQ, Michel (2019) Sérotonine. Paris : Flammarion.

21 L'écrivain considère lui-même avoir beaucoup évolué en la matière : "Votre sujet est bon, mais à mon avis ce serait une erreur de le restreindre à La Possibilité d'une île, parce que j'ai la sensation d'avoir pas mal évolué » (courriel du 28 août 2015, voir : Jafuel 2016 : 15).

22 Le présent article a été élaboré dans le cadre du programme de recherche n ${ }^{\circ} 6-0265$ consacré aux recherches littéraires interculturelles financé par des fonds publics gérés par l'Agence nationale de la recherche de la République de Slovénie. 


\section{Traductions slovènes}

HOUELLEBECQ, Michel (2000) Osnovni delci. Trad. Mojca Medvedšek. Ljubljana : Cankarjeva založba. [Dans l'article : Houellebecq - Medvedšek 2000.]

HOUELLEBECQ, Michel (2004) Razširitev področja boja. Trad. Marko Trobevšek. Ljubljana : Modrijan. [Dans l'article : Houellebecq - Trobevšek 2004.]

HOUELLEBECQ, Michel (2020) Serotonin. Trad. Iztok Ilc. Ljubljana : Cankarjeva založba. [Dans l'article : Houellebecq - Ilc. 2020.]

\section{Secondaire}

AUTHIER-REVUZ, Jacqueline (1978) « Les formes du discours rapporté. Remarques syntaxiques et sémantiques à partir des traitements proposés ». Documentation et recherche en linguistique allemande contemporain - Vincennes, $\mathrm{n}^{\circ}$ 17, 1978, pp. 1-87

AUTHIER-REVUZ, Jacqueline (1992-1993) « Repères dans le champ du discours rapporté », L'Information grammaticale, $\mathrm{n}^{\circ} 55,38-42$ et n ${ }^{\circ} 56$, pp. 10-15.

AUTHIER-REVUZ, Jacqueline (1996) « Remarques sur la catégorie de l'îlot textuel». Cahiers du français contemporain, $\mathrm{n}^{\circ} 3$, pp. 91-115.

AUTHIER-REVUZ, Jacqueline (2019) « Autonymie: pièges et stratégies d'une énonciation à doubles-fonds ». M.-F. Marein (et al.) [dir.], Les Illusions de l'autonymie. La parole rapportée de l'autre dans la littérature. Paris : Hermann, pp. 15-39.

[Coll.] (2002) Lexique des règles typographiques en usage à l'Imprimerie nationale. Paris : Imprimerie nationale.

COULEAU, Christèle (2013) « 'Les âmes moyennes'. De la trivialité comme poétique romanesque ». Bruno Viard, Sabine Van Wesemael [dir.], L'Unité de l'œuvre de Michel Houellebecq, Paris : Classiques Garnier, p. 13-26.

ESTIER, Samuel (2015) À propos du « style » de Houellebecq. Retour sur une controverse (1998-2010). Lausanne: Archipel Essais.

JAFUEL, Laure (2016) L'italique dans La Possibilité d'une île de Michel Houellebecq, mémoire de master. Metz : Université de Lorraine.

HERSCHBERG PIERROT, Anne (1993) Stylistique de la prose. Paris : Belin.

MICHEL, Jacqueline (1989) Une mise en récit du silence, Le Clézio /Bosco/ Gracq. Paris : José Corti.

NOGUEZ, Dominique (2003) Houellebecq, en fait, Paris : Fayard.

PERKO, Gregor (2012) « Modalisation autonymique et dynamisme communicatif ». Écho des études romanes, VIII/1, pp. 255-264.

REY-DEBOVE, Josette (1997) Le Métalangage. Paris : Armand Colin.

RIEGEL, Martin [et al.] (1994) La Grammaire méthodique du français. Paris : PUF.

VAN WESEMAEL Sabine (2013) « Michel Houellebecq : un auteur postréaliste ». B. Viard, S. van Wesemael (dir.), L'Unité de l'œuvre de Michel Houellebecq, Paris. Classiques Garnier, pp. 325-336. 
POVZETEK

\section{PREVAJANJE AVTONIMNIH KONOTACIJ V TREH ROMANIH MICHELA HOUELLEBECQA}

V članku avtorica raziskuje obravnavo t. i. avtonimnih elementov oz. razširjene, po pravopisu nedoločene rabe kurzivne pisave in narekovajev v slovenskih prevodih treh romanov M. Houellebecqa, kot so jih izoblikovali trije različni slovenski prevajalci. Po uvodni opredelitvi so analizirani elementi razvrščeni v tri poglavitne kategorije, glede na to, ali posredujejo govorico določenih ali nedoločenih posameznikov, samega pripovedovalca ali celotne družbe. V okviru sloga, ki ga pogosto označujejo kot »belega«, razširjena uporaba ležeče pisave in narekovajev s funkcijo avtonimne konotacije omogoča posredovanje zelo finih pomenskih odtenkov in znatno obogati avtorjevo besedilo. Enako kot metaforične mreže tudi slogemi, kot je ponavljajoča se in razširjena uporaba ležeče pisave in narekovajev, od prevajalca zahtevajo predhodno delo interpretacije in razvoj koherentne strategije, ki omogoča ohranitev njihove izvorne funkcije v pripovedi. Čeprav lahko analizirane prevode štejemo za globalno zveste Houellebecqovemu besedilu in so omogočili, da je pisatelj v Sloveniji postal zelo znan, je jasno, da nobenega od njih ne odlikuje stroga in koherentna obravnava avtonimnih konotacij, na katere kaže razširjena uporaba kurzivne pisave in narekovajev. Najpogostejše in najbolj škodljive anomalije za fino razumevanje besedila so vzdrževanje teh vizualnih znakov tam, kjer ni nujno, ali nasprotno njihova neuporaba tam, kjer jih je povsem mogoče in primerno vzdrževati. Avtorica sproti opozarja na možne rešitve in zagovarja izoblikovanje dosledne prevodoslovne strategije pri obravnavi analiziranih elementov.

Ključne besede: Houellebecq, avtonimni elementi, kurzivna pisava, sodobni francoski roman, traduktologija, literarno prevajanje, prevajanje iz francoščine v slovenščino

\section{ABSTRACT}

\section{TRANSLATION OF AUTONYMIC CONNOTATIONS IN THREE NOVELS BY MICHEL HOUELLEBECQ}

This paper deals with the autonyms or the widespread, orthographically indeterminate use of italics and quotation marks in Slovene translations of three of Houellebecq's novels, which have been elaborated by three different Slovene translators. Autonyms are divided into three main categories according to whether they convey the words of definite or indefinite individuals, the language of the narrator himself or the concepts and values of the whole society. Within the framework of a style often qualified as "white", the extended use of italics and quotation marks with function of autonymic connotation allows the author to transmit to the reader very fine semantic nuances and contributes to the richness of his work. Like metaphorical networks, stylems such as the recurrent 
and extended use of italics and quotation marks require from the translator a preliminary work of interpretation and the development of a coherent strategy making it possible to preserve intact the function of these elements in the narrative. Although the three translations analysed can be considered as globally faithful to Houellebecq's texts and made possible the notoriety of the French writer in Slovenia, none of them is distinguished by a rigorous and coherent treatment of autonymic connotations indicated by non-normative use of italics and quotation marks. The most frequent and damaging anomalies are the maintenance of these visual signs where the expressions indicated in French do not have to be in Slovenian or, on the contrary, their elimination where it is perfectly possible and appropriate to maintain them for the detailed understanding of the text. The author draws attention to possible solutions and opts for the elaboration of a consistent translation strategy for the treatment of the analysed elements.

Keywords: Houellebecq, autonyms, italic, contemporary French novel, translatology, literary translation, translation from French to Slovene 